PROCEEDINGS OF THE

AMERICAN MATHEMATICAL SOCIETY

Volume 128, Number 8, Pages 2223-2232

S 0002-9939(99)05230-2

Article electronically published on November 29, 1999

\title{
TILTING UP ITERATED TILTED ALGEBRAS
}

\author{
IBRAHIM ASSEM, DIETER HAPPEL, AND SONIA TREPODE \\ (Communicated by Ken Goodearl)
}

\begin{abstract}
We show that, if $A$ is a representation-finite iterated tilted algebra of euclidean type $Q$, then there exist a sequence of algebras $A=A_{0}, A_{1}, A_{2}, \ldots$, $A_{m}$, and a sequence of modules $T_{A_{i}}^{(i)}$, where $0 \leq i<m$, such that each $T_{A_{i}}^{(i)}$ is an APR-tilting $A_{i}$-module, or an APR-cotilting $A_{i}$-module, End $T_{A_{i}}^{(i)}=A_{i+1}$ and $A_{m}$ is tilted representation-finite.
\end{abstract}

\section{INTRODUCTION}

Throughout this paper, we let $k$ denote a fixed algebraically closed field. By algebra is always meant a finite dimensional associative $k$-algebra with an identity, which we assume moreover to be basic and connected, and by module is meant a finitely generated right $A$-module. Tilting theory is by now an established tool in the representation theory of algebras. In particular, the classes of tilted and iterated tilted algebras, introduced by means of the tilting process, were very useful, for instance, in the classification of the self-injective algebras of polynomial growth (see [1, 7], [11]). We recall their definition. Let $Q$ be a finite, connected quiver without oriented cycles and let $k Q$ denote the path algebra of $Q$. An algebra $A$ is said to be iterated tilted of type $Q$ if there exists a going-up tilting series from $A$ to $k Q$, that is, a sequence of algebras $A=A_{0}, A_{1}, \ldots, A_{m}=k Q$ and a sequence of tilting modules $T_{A_{i}}^{(i)}$, where $0 \leq i<m$, such that $\operatorname{End} T_{A_{i}}^{(i)}=A_{i+1}$ and each $T_{A_{i}}^{(i)}$ is separating, that is, such that each indecomposable $A_{i}$-module $M$ satisfies either $\operatorname{Hom}_{A_{i}}\left(T^{(i)}, M\right)=0$ or $\operatorname{Ext}_{A_{i}}^{1}\left(T^{(i)}, M\right)=0$ (see [2]). This implies that, if $A_{i}$ is representation-finite, then so is $A_{j}$ for each $0 \leq j \leq i$. If $m \leq 1$, then $A$ is said to be a tilted algebra of type $Q$.

In order to study the representation-finite iterated tilted algebras of euclidean type, O. Roldán (private communication) has formulated the following conjecture which would allow to reduce their study to that of the representation-finite tilted algebras of the same type. Let $Q$ be a euclidean quiver, and $A$ be a representationfinite iterated tilted algebra of type $Q$. The conjecture states that there exists a sequence of tilts $A=A_{0}, A_{1}, \ldots, A_{m-1}, A_{m}=k Q$ as in the definition above, but with $A_{m-1}$ representation-finite [1] (6.5). We show that the conjecture, in this form, is not true (see example (3.4) (a)), so that separating tilting modules do not suffice to reduce the study of iterated tilted algebras of euclidean type

Received by the editors December 15, 1997 and, in revised form, September 10, 1998.

2000 Mathematics Subject Classification. Primary 16G60, 16G20.

Key words and phrases. Representation-finite iterated tilted algebras of euclidean type, APRtilting and cotilting modules, derived category.

(C)2000 American Mathematical Society 
to that of representation-finite tilted algebras of the same type. The objective of this paper is to show that the use of both separating tilting and separating cotilting modules (even, of a particularly nice type) will achieve that goal. Namely, replacing the separating tilting modules by APR-tilting or APR-cotilting modules (for the definitions, see (2.1)), one obtains the following statement.

Theorem. Let $Q$ be a euclidean quiver without oriented cycles, and $A$ be a representation-finite iterated tilted algebra of type $Q$. Then there exist a sequence of algebras $A=A_{0}, A_{1}, \ldots, A_{m}$, and a sequence of modules $T_{A_{i}}^{(i)}$, where $0 \leq i<m$, such that each $T_{A_{i}}^{(i)}$ is an APR-tilting $A_{i}$-module, or an APR-cotilting $A_{i}$-module, End $T_{A_{i}}^{(i)}=A_{i+1}$ and $A_{m}$ is tilted representation-finite.

Further, we show that this theorem is not true if one allows $Q$ to be a wild quiver instead of a euclidean quiver (see Example (3.4) (b)).

The case where the underlying graph of $Q$ is the euclidean diagram $\tilde{\mathbb{A}}_{n}$ was considered by the third author in 12, 13, using the classification of the tilted and iterated tilted algebras of type $\tilde{\mathbb{A}}_{n}$ (see [3], [10]). The purpose of the present paper is to provide a proof for the general case that does not use any classification result.

The paper is organised as follows. In section 2, after setting the notation and briefly recalling some definitions and results that will be needed in the sequel, we prove a necessary and sufficient condition for an iterated tilted algebra of euclidean type to be representation-finite, then a lemma on almost complete tilting modules over tame hereditary algebras. Section 3 consists of two lemmata followed by the proof of our theorem and ends with the aforementioned examples.

\section{Preliminaries}

2.1. Notation. Let $A$ be a finite dimensional basic and connected $k$-algebra. We denote by $Q_{A}$ the ordinary quiver of $A$, and by $\left(Q_{A}\right)_{0}$ the set of points of $Q_{A}$. For a point $a \in\left(Q_{A}\right)_{0}$, we denote by $S(a)$ the corresponding simple $A$-module, and by $P(a)$ the projective cover of $S(a)$. We use freely, and without further reference, facts about the module category $\bmod A$ and the Auslander-Reiten translations $\tau_{A}=\mathrm{DTr}$ and $\tau_{A}^{-1}=\operatorname{TrD}$, as in [5], [9].

An $A$-module $T$ is called a tilting (or cotilting) module if $\operatorname{pd} T_{A} \leq 1$ (or $\operatorname{id} T_{A} \leq 1$, respectively), $\operatorname{Ext}_{A}^{1}(T, T)=0$ and the number of isomorphism classes of indecomposable summands of $T$ equals the number of points in $Q_{A}$. Given a tilting module $T_{A}$, there exists a close connection between the representation theories of $A$ and of End $T_{A}$ : this connection is known as tilting theory, and we refer the reader to [1, 7] for the details. A tilting module $T_{A}$ is called separating if, for each indecomposable $A$-module $M$, we have either $\operatorname{Hom}_{A}(T, M)=0 \operatorname{or}_{E^{\prime}} \operatorname{Ex}_{A}^{1}(T, M)=0$. If $T_{A}$ is a separating tilting module, then the almost split sequences in $\bmod A$ are totally determined by those in $\bmod \operatorname{End} T_{A}$. An example of separating tilting module is provided by the so-called APR-tilting modules: let $P(a)_{A}$ be a simple projective module which is not injective; then the module $\tau_{A}^{-1} P(a) \oplus\left(\bigoplus_{b \neq a} P(b)\right)$ is the corresponding APR-tilting module. Separating cotilting modules and APR-cotilting modules are defined dually.

2.2. The derived category. Let $A$ be an algebra. We denote by $D^{b}(\bmod A)$ the derived category of bounded complexes of $A$-modules. The study of $D^{b}(\bmod A)$ is 


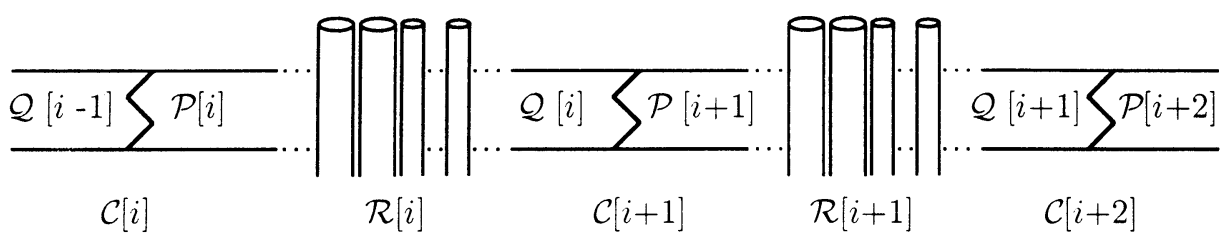

FiguRE 1.

especially fruitful if $A$ is an iterated tilted algebra, as is seen from the following theorem [7] (IV.5.4), p. 176.

Theorem. Let $Q$ be a finite connected quiver without oriented cycles. An algebra $A$ is iterated tilted of type $Q$ if and only if there exists an equivalence of triangulated categories $D^{b}(\bmod A) \cong D^{b}(\bmod k Q)$.

Further, the structure of $D^{b}(\bmod k Q)$ is known 7 (I.5.5) p. 54. If, in particular, $Q$ is a euclidean quiver, then the Auslander-Reiten quiver of $k Q$ consists of a component $\mathcal{P}$ containing the projective $k Q$-modules, a component $\mathcal{Q}$ containing the injective $k Q$-modules, and a stable tubular family $\mathcal{R}=\left(\mathcal{R}_{\lambda}\right)_{\lambda \in \mathbb{P}_{1}(k)}$ separating $\mathcal{P}$ from $\mathcal{Q}$ (see [6], [9]). The quiver of $D^{b}(\bmod k Q)$ may then be visualised as shown in Figure 1, where each $\mathcal{P}[i]$ (or $\mathcal{Q}[i], \mathcal{R}[i]$ ) is a copy of $\mathcal{P}$ (or $\mathcal{Q}, \mathcal{R}$, respectively) indexed by $i \in \mathbb{Z}$. The components $\mathcal{C}[i]$ are referred to as the transjective components, and the $\mathcal{R}[i]$ as the regular components.

Let $A$ be an iterated tilted algebra of type $Q$. The image of the module $A_{A}$ under the composition of the embedding of $\bmod A$ inside $D^{b}(\bmod A)$ with the triangleequivalence $F: D^{b}(\bmod A) \cong D^{b}(\bmod k Q)$ is a tilting complex $T^{\bullet}=F\left(A_{A}\right)$ (in the sense of [8]) which we call the standard complex. For a point $a \in\left(Q_{A}\right)_{0}$, we write $T^{\bullet}(a)=F P(a)$. Thus, $T^{\bullet}=\underset{a \in\left(Q_{A}\right)_{0}}{\bigoplus^{\bullet}(a)}$.

2.3. Proposition. Let $A$ be an iterated tilted algebra of euclidean type $Q$, and let $F: D^{b}(\bmod A) \rightarrow D^{b}(\bmod k Q)$ be a triangle-equivalence. Then $A$ is representationfinite if and only if there exist two points $a \neq b$ in $Q_{A}$, and two integers $i_{a} \neq i_{b}$ such that $T^{\bullet}(a) \in \mathcal{C}\left[i_{a}\right]$ and $T^{\bullet}(b) \in \mathcal{C}\left[i_{b}\right]$.

Proof. Since the sufficiency is just part (a) of [7] (IV.7.1), p. 188, we only show the necessity. We assume that the stated condition does not hold, and prove that this implies that $A$ is representation-infinite.

We first notice that there exists at least one point $a \in\left(Q_{A}\right)_{0}$ such that $T^{\bullet}(a)$ lies in a transjective component : indeed, if this is not the case, then the indecomposable summands $T^{\bullet}(x)$ of the standard complex $T^{\bullet}$ all belong to the regular components, which would imply that the vectors $\underline{\operatorname{dim}} T^{\bullet}(x)$ are linearly dependent, a contradiction to the fact that they form a basis in $K_{0}\left(D^{b}(\bmod k Q)\right)$, by [7] (III.1.2), p. 96 . Therefore the assumption that the stated condition does not hold implies that the indecomposable summands of the standard complex lie in at most one transjective component $\mathcal{C}[i]$, say. The standard complex $T^{\bullet}$ decomposes thus as $T^{\bullet}=T^{\mathbf{\bullet}} \oplus T^{\prime \prime}$, where $T^{\mathbf{\bullet}} \in \mathcal{C}[i]$ is non-zero, while the indecomposable summands of $T^{\prime \prime}$ are all regular.

Let $X^{\bullet} \in \mathcal{R}[i]$ be any indecomposable complex that is regular and homogeneous (that is, such that $\left.\tau_{D^{b}(\bmod k Q)} X^{\bullet} \cong X^{\bullet}\right)$. Then we claim that $X^{\bullet}$ belongs to the image $F(\bmod A)$ of $\bmod A$ inside $D^{b}(\bmod k Q)$. This implies the 
conclusion, because there exist infinitely many isomorphism classes of regular homogeneous complexes in $\mathcal{R}[i]$. By [7] (IV.5.1), p. 175, it suffices to show that $\operatorname{Hom}_{D^{b}(\bmod k Q)}\left(T^{\bullet}, X^{\bullet}[j]\right)=0$ for all $j \neq 0$. Now, if $Y^{\bullet}$ is an indecomposable summand of $T^{\prime \prime} \bullet$, then $\operatorname{Hom}_{D^{b}(\bmod k Q)}\left(Y^{\bullet}, Y^{\bullet}[1]\right)=0$ (because $T^{\bullet}$ is a tilting complex); hence $Y^{\bullet}$ cannot be homogeneous. This shows that all the indecomposable summands of $T^{\prime \prime} \bullet$ that lie in $\mathcal{R}[i]$ belong to tubes distinct from, and hence orthogonal to, the one containing $X^{\bullet}$. Our claim then follows from the structure of the morphisms in $D^{b}(\bmod k Q)$.

2.4. The following lemma will be useful in the final step of the proof of our main result. We recall that, if $A$ is an algebra, then a module $T_{A}$ is an almost complete tilting module if there exists an indecomposable module $X$ such that $T \oplus X$ is a tilting module. For the representation theory of tame hereditary algebras, we refer the reader to [6], [9].

Lemma. Let $H$ be a tame hereditary algebra, and $T=T^{\prime} \oplus T^{\prime \prime}$ be an almost complete tilting module, with $T^{\prime}$ indecomposable projective and $T^{\prime \prime}$ regular. Then there exists, up to isomorphism, at most one indecomposable summand $T_{0}^{\prime \prime}$ of $T^{\prime \prime}$ on the mouth of each tube such that $\operatorname{Hom}_{H}\left(T^{\prime}, T_{0}^{\prime \prime}\right) \neq 0$.

Proof. By tilting, we may assume that the quiver $Q_{H}$ of $H$ has the orientation in the Tables of [6]. If the underlying graph of $Q_{H}$ is $\tilde{\mathbb{A}}_{n}$, then the statement follows from [4] (3.5). The cases where the underlying graph is $\tilde{\mathbb{E}}_{6}, \tilde{\mathbb{E}}_{7}, \tilde{\mathbb{E}}_{8}$ are easily done by inspection, using the Tables of [6]. We thus only need to consider the case when the underlying graph of $Q_{H}$ is $\tilde{\mathbb{D}}_{n}$ with the shown orientation.

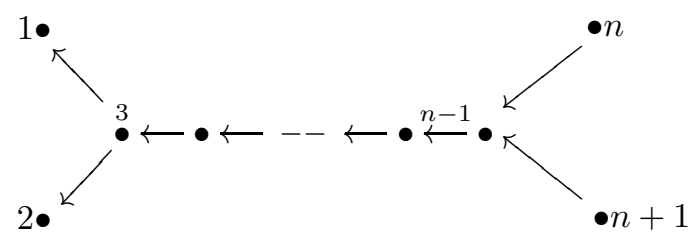

Since $T$ is almost complete, it has $n$ isomorphism classes of indecomposable summands, of which $n-1$ are regular. There is therefore one indecomposable summand of $T$ in each of the two tubes of rank 2 , and $n-3$ in the tube of rank $n-2$. Since the statement is clear for tubes of rank 2 (and even 3 ), we consider the tube $\mathcal{T}$ of rank $n-2$ and assume $n \geq 6$. By the Tables of [6], the mouth of $\mathcal{T}$ contains a sincere indecomposable $M$ with $\underline{\operatorname{dim}} M={ }_{1}^{1} 11 \ldots 11_{1}^{1}$, and the simple $H$-modules $S(i)$, where $3 \leq i \leq n-1$. Assume $T^{\prime}=P(j)$. If $j \in\{1,2, n, n+1\}$, then the only module on the mouth of $\mathcal{T}$ to which $P(j)$ maps is $M$ and we are done. If not, then $P(j)$ maps exactly to two modules on the mouth of $\mathcal{T}$, namely $M$ and $S(j)$. Assume that both $M$ and $S(j)$ are summands of $T^{\prime \prime}$. Since, for each indecomposable summand $X$ of $T^{\prime \prime}$, we have $\operatorname{Hom}_{H}(P(j), \tau X) \cong D \operatorname{Ext}_{H}^{1}(X, P(j))=0$, the module $\tau X$ is not sincere. Consequently, $X$ lies in the wing $\mathcal{W}$ determined by the mouth modules $S(n-2), S(n-3), \ldots, S(3), M$. The mesh category of $\mathcal{W}$ is isomorphic to the mesh category of the Auslander-Reiten quiver of a hereditary algebra $H^{\prime}$ of type $\mathbb{A}_{n-3}$ with linear orientation. Since $T^{\prime \prime}$ has $n-3$ indecomposable summands, it maps under this isomorphism onto a tilting $H^{\prime}$-module. However, the unique projective-injective $H^{\prime}$-module must be a summand of any tilting module. This implies that the corresponding module $N$ in $\mathcal{W}$ (where $\underline{\operatorname{dim}} N={ }_{1}^{1} 22 \ldots 21_{1}^{1}$ ) is a 
summand of $T^{\prime \prime}$. But $\underline{\operatorname{dim}} \tau N={ }_{0}^{0} 11 \ldots 11_{0}^{0}$ and, in particular, $\operatorname{Ext}_{H}^{1}(N, P(j)) \cong$

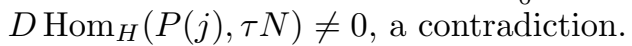

\section{The MAin Result}

3.1. We shall use essentially the following lemma and its dual (which we do not state for the sake of brevity). They assert that, under the action of an APR-tilting (or cotilting) module, the images of the points of $\bmod A$ under the triangle-equivalence $D^{b}(\bmod A) \cong D^{b}(\bmod k Q)$ get closer together.

Lemma. Let $A$ be an iterated tilted algebra of type $Q$, and let $F: D^{b}(\bmod A) \rightarrow$ $D^{b}(\bmod k Q)$ be a triangle-equivalence. Let $P_{A}$ be a simple projective module, and $P_{A}^{\prime}$ be such that $A_{A}=P \oplus P^{\prime}$. Then:

(a) If id $P=1$, then $F\left(\tau_{A}^{-1} P\right) \cong \tau_{D^{b}(\bmod k Q)}^{-1} F(P)$.

(b) If id $P>1$, then $\operatorname{Hom}_{D^{b}(\bmod k Q)}\left(F\left(\tau_{A}^{-1} P\right), F\left(P^{\prime}\right)\right) \neq 0$.

Proof. (a) We have an almost split sequence

$$
0 \rightarrow P \rightarrow E \rightarrow \tau_{A}^{-1} P \rightarrow 0
$$

where $\operatorname{pd} \tau_{A}^{-1} P \leq 1$ and $\operatorname{id} P=1$. By [7] (I.4.7), p.37, we have an almost split triangle in $D^{b}(\bmod A)$

$$
P \rightarrow E \rightarrow \tau_{A}^{-1} P \rightarrow P[1]
$$

Hence, applying $F$, we get an almost split triangle in $D^{b}(\bmod k Q)$

$$
F P \rightarrow F E \rightarrow F\left(\tau_{A}^{-1} P\right) \rightarrow F P[1] .
$$

In particular, $F\left(\tau_{A}^{-1} P\right) \cong \tau_{D^{b}(\bmod k Q)}^{-1} F(P)$.

(b) If id $P \geq 2$, then $\operatorname{Hom}_{A}\left(\tau_{A}^{-1} P, A\right) \neq 0$. Since $\operatorname{Hom}_{A}\left(\tau_{A}^{-1} P, P\right)=0$, then $\operatorname{Hom}_{A}\left(\tau_{A}^{-1} P, P^{\prime}\right) \neq 0$. Applying $F$ yields $\operatorname{Hom}_{D^{b}(\bmod k Q)}\left(F\left(\tau_{A}^{-1} P\right), F P^{\prime}\right) \neq 0$.

3.2. Lemma. Let $A$ be a representation-finite iterated tilted algebra of euclidean type $Q$ such that any APR-tilting module tilts $A$ to a representation-infinite algebra. Then there exist exactly two consecutive transjective components $\mathcal{C}[t]$ and $\mathcal{C}[t+1]$ that contain summands of the standard complex, and furthermore, $\bigcup_{j<t} \mathcal{R}[j]$ contains no summand of the standard complex.

Proof. By (2.3), there exist $t \neq s$ in $\mathbb{Z}$ such that $\mathcal{C}[t]$ and $\mathcal{C}[s]$ contain summands of the standard complex. We may assume that $t<s$ and that $\mathcal{C}[r]$ contains no summand of the standard complex if $r<t$ or $r>s$.

Assume that there exists $a \in\left(Q_{A}\right)_{0}$ such that $T^{\bullet}(a) \in \bigcup_{j<t} \mathcal{R}[j]$. Then there exist a sink $a \in\left(Q_{A}\right)_{0}$ and an integer $j<t$ such that $T^{\bullet}(a) \in \mathcal{R}[j]$. The endomorphism algebra of the APR-tilting module at $a$ is clearly iterated tilted. It is moreover representation-finite, by $(2.3)$, since $\mathcal{C}[t] \neq \mathcal{C}[s]$ contain summands of its standard complex. This contradicts our hypothesis on $A$, and shows that $\bigcup_{j<t} \mathcal{R}[j]$ contains no summand of the standard complex.

We claim that $s=t+1$. If this is not the case, then $s>t+1$ and, by hypothesis, there exists a sink $a \in\left(Q_{A}\right)_{0}$ such that $T^{\bullet}(a) \in \mathcal{C}[t]$. Then $T^{\bullet}(a)$ is the unique indecomposable summand of $T^{\bullet}=F\left(A_{A}\right)$ to lie in $\mathcal{C}[t]$ (otherwise, considering the APR-tilting module at $a$ yields a contradiction, as above). On the other hand, 
there is no indecomposable summand of $T^{\bullet}$ in $\mathcal{C}[t+1]$ (otherwise, consider again the APR-tilting module at $a)$. We shall show that $F\left(\tau_{A}^{-1} P(a)\right) \in \mathcal{C}[t+1]$. This will imply the statement since, considering the endomorphism algebra of the APRtilting module at $a$, there exist summands of its standard complex in $\mathcal{C}[t+1]$ and $\mathcal{C}[s]$, again a contradiction.

The almost split sequence $0 \rightarrow P(a) \rightarrow E \rightarrow \tau_{A}^{-1} P(a) \rightarrow 0$ induces a (not necessarily almost split) triangle in $D^{b}(\bmod A)$

$$
P(a) \stackrel{u}{\longrightarrow} E \stackrel{v}{\longrightarrow} \tau_{A}^{-1} P(a) \stackrel{w}{\longrightarrow} P(a)[1]
$$

such that $u \neq 0, v \neq 0$ and $w \neq 0$. Applying $F$ yields a triangle in $D^{b}(\bmod k Q)$

$$
F P(a) \stackrel{F u}{\longrightarrow} F E \stackrel{F v}{\longrightarrow} F\left(\tau_{A}^{-1} P(a)\right) \stackrel{F w}{\longrightarrow} F P(a)[1] .
$$

Since $F u \neq 0$ and $E_{A}$ is projective, the considerations above show that $F E \in \mathcal{R}[t]$.

Taking a complete slice in $\mathcal{C}[t]$ having $T^{\bullet}(a)=F P(a)$ as a unique source, we get a hereditary algebra $H$ such that we have a triangle-equivalence $D^{b}(\bmod A) \cong$ $D^{b}(\bmod H)$ under which $F P(a)$ maps onto the unique simple projective $H$-module. On the other hand, $F E$ maps into the regular component of $\bmod H$. Since we can assume that $H=k Q$, we can (and will) identifiy $F P(a), F E$, etc. to their images. Since $F P(a)$ is simple, $F u$ is a monomorphism. Hence we have a diagram

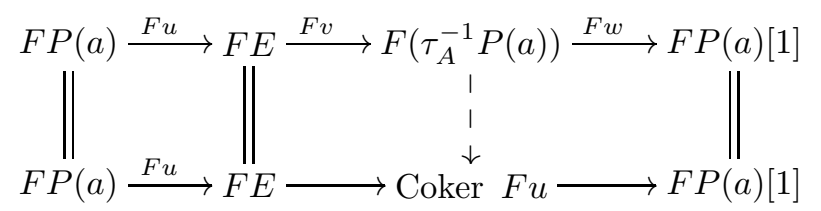

where the dotted map exists and is an isomorphism by the elementary properties of triangulated categories [7] (I.1.1), p.2. Thus Coker $F u \cong F\left(\tau_{A}^{-1} P(a)\right)$. Now Coker $F u$ is not regular, since, otherwise, the regularity of $F E$ implies that $F P(a)$ is also regular (because the regular $H$-modules form an abelian subcategory of $\bmod H)$. Since $\operatorname{Hom}_{D^{b}(\bmod H)}($ Coker $F u, F P(a)[1]) \neq 0$, we deduce that Coker $F u \in \mathcal{C}[t+1]$.

Proof of the theorem. Let $A$ be a representation-finite iterated tilted algebra of euclidean type $Q$. By applying a finite sequence of APR-cotilting modules, we obtain, by [7] (IV.7.4), p. 190, an iterated tilted algebra $B$ of type $Q$ having the following property : $B$ is representation-finite, but the endomorphism algebra of any APR-cotilting $B$-module is representation-infinite. Clearly, we may assume that $A=B$.

By (2.3), there exist $t \neq s$ in $\mathbb{Z}$ such that $\mathcal{C}[t]$ and $\mathcal{C}[s]$ contain summands of the standard complex $T^{\bullet}=F A_{A}$, where $F$ denotes as usual a triangle equivalence $D^{b}(\bmod A) \rightarrow D^{b}(\bmod k Q)$. We may assume that $0=t<s$ and that $\mathcal{C}[r]$ contains no summand of $T^{\bullet}$ for $r<0$ and $r>s$. By the dual of (3.2), we have further that $s=1$ and $\bigcup_{j>1} \mathcal{R}[j]$ contains no summand of the standard complex.

We thus know that $\mathcal{C}[0]$ and $\mathcal{C}[1]$ contain indecomposable summands of $T^{\bullet}$ and that $\bigcup_{j \leq 0} \mathcal{R}[j]$ may. We now show that we may assume that no summand of $T^{\bullet}$ 
lies in $\bigcup_{j<0} \mathcal{R}[j]$. If this is the case, then there exist a sink $a \in\left(Q_{A}\right)_{0}$ and $j<0$ such that $T^{\bullet}(a)=F P(a) \in \mathcal{R}[j]$. Let $b \in\left(Q_{A}\right)_{0}$ be such that $T^{\bullet}(b)=F P(b) \in \mathcal{C}[1]$. The endomorphism algebra of the APR-tilting module at $a$ is still representationfinite (by (2.3)) and there exists a path from $F\left(\tau_{A}^{-1} P(a)\right)$ to $F P(b)$. Applying successively a sequence of APR-tilting modules corresponding to those summands of $T^{\bullet}$ lying in $\bigcup_{j<0} \mathcal{R}[j]$, and using (3.1), we thus reach an algebra $C$ having the following properties :

(i) $C$ is representation-finite, and

(ii) $F C_{C} \in \mathcal{C}[0] \cup \mathcal{R}[0] \cup \mathcal{C}[1]$, where $F$ denotes, as usual, a triangle-equivalence $F: D^{b}(\bmod C) \rightarrow D^{b}(\bmod k Q)$.

We now claim that $C$ can be reduced (by applying a finite sequence of APRtilting or APR-cotilting modules) to either a representation-finite tilted algebra and then we are done - or else to the case when the standard complex $F C_{C}=T^{\bullet}$ has a unique indecomposable summand $T_{0}^{\bullet}$ in $\mathcal{C}[0]$ and a unique one $T_{1}^{\bullet}$ in $\mathcal{C}[1]$.

Assume that the latter situation does not occur. In particular, $T^{\bullet}=F C_{C}$ has more than one indecomposable summand in $\mathcal{C}[0]$ or $\mathcal{C}[1]$, say the former. Let $T_{0}^{\bullet}$ denote a "last" indecomposable summand of $T^{\bullet}$ to lie in $\mathcal{C}[0]$, that is, let $T^{\bullet}$ be such that there exists no path in $\mathcal{C}[0]$ from $T_{0}^{\bullet}$ to another indecomposable summand of $T^{\bullet}$. Taking a complete slice in $\mathcal{C}[0]$ having $T_{0}^{\bullet}$ as unique source yields a hereditary algebra $H$ such that $D^{b}(\bmod C) \cong D^{b}(\bmod H)$. We may assume that the sequence of APR-tilting and cotilting modules used to reach the algebra $C$ has the property that the number of isomorphism classes of indecomposable summands of $T^{\bullet}$ lying outside $\bmod H$ is the least possible. It follows from (3.1) that there exists a finite sequence of APR-tilting modules transforming $C$ to $D$ (say) such that there exists a sink $a \in\left(Q_{D}\right)_{0}$ with $T^{\bullet}(a)$ not in $\bmod H$, but the middle term $E$ of the almost split sequence

$$
0 \rightarrow P(a) \rightarrow E \rightarrow \tau_{D}^{-1} P(a) \rightarrow 0
$$

has none of its indecomposable summands lying in $\bmod H$. By an argument similar to the one used in (3.2), we deduce that $F\left(\tau_{D}^{-1} P(a)\right)$ lies in $\bmod H$, and this contradicts the assumed minimality. This completes the proof of our claim.

We note that, if all the indecomposable summands of $T^{\bullet}=F C_{C}$ lie inside $\bmod H$, then the tilting complex $T^{\bullet}$ is a tilting module and $C$ is a tilted algebra.

We now consider the case where $F C_{C}$ has a unique indecomposable summand $T_{0}^{\bullet}$ in $\mathcal{C}[0]$ and a unique one $T_{1}^{\bullet}$ in $\mathcal{C}[1]$. Clearly, this implies that $T^{\bullet}=F P(a)$, with $a$ a sink in $Q_{C}$ and $T_{1}^{\bullet}=F P(b)$, with $b$ a source.

We claim that we may assume $a$ to be the only sink, and $b$ the only source in $Q_{C}$. Indeed, if $a^{\prime} \neq a$ is another sink in $Q_{C}$, then $T^{\bullet}\left(a^{\prime}\right)$ necessarily lies in $\mathcal{R}[0]$. We claim that $F \tau_{C}^{-1} P\left(a^{\prime}\right) \in \mathcal{R}[0]$. Indeed, by (3.1), either id $P\left(a^{\prime}\right)_{C}=1$, and then $F \tau_{C}^{-1} P\left(a^{\prime}\right)=\tau_{D^{b}(\bmod H)}^{-1} F P\left(a^{\prime}\right) \in \mathcal{R}[0]$, or else id $P\left(a^{\prime}\right)_{C}>1$, and then $\operatorname{Hom}_{D^{b}(\bmod H)}\left(F \tau_{C}^{-1} P\left(a^{\prime}\right), F P^{\prime}\right) \neq 0$, where $P^{\prime}=\bigoplus_{c \neq a^{\prime}} P(c)$. Consequently, there exists a path $F \tau_{C}^{-1} P\left(a^{\prime}\right) \rightarrow \cdots \rightarrow T_{1}^{\bullet}$ in $D^{b}(\bmod H)$. Therefore the existence of a morphism $F E \rightarrow F \tau_{C}^{-1} P\left(a^{\prime}\right)$ implies that $T_{1}^{\bullet}$ cannot be a summand of $F E$. Thus $F E \in \mathcal{R}[0]$. Since $\mathcal{R}[0]$ is abelian, we deduce, as in (3.2), that $F \tau_{C}^{-1} P\left(a^{\prime}\right) \in \mathcal{R}[0]$, as required. 
We now consider the regular summands of $T^{\bullet}$, lying in $\mathcal{R}[0]$. We recall that the endomorphism algebra of a partial tilting module lying in a tube of a tame hereditary algebra is a direct product of tilted algebras of type $\mathbb{A}_{n}$ (see, for instance, [4] (1.4)). This first implies that the summands of $T^{\bullet}$ belonging to a given tube may be assumed to lie on a sectional path : indeed, if this is not the case, then it follows from (2.4) that $Q_{C}$ would have a source or a sink distinct from $a$ or $b$, a contradiction. Next, those summands of $T^{\bullet}$ belonging to a given tube are equal to all exceptional points on the sectional path on which they lie : this is because a euclidean diagram has $n+1$ points; hence $T^{\bullet}=F C_{C}$ has $(n+1)-2=$ $n-1$ summands in $\mathcal{R}[0]$, and an indecomposable summand of $T^{\bullet}$ is the image of an exceptional $H$-module under the embedding of $\bmod H$ into $D^{b}(\bmod H)$. This argument also shows that the endomorphism algebra of the direct sum of those summands of $T^{\bullet}$ that lie in $\mathcal{R}[0]$ is the direct product of (at most three) hereditary Nakayama algebras $H_{i}$ of quiver

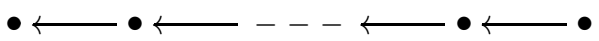

Now, since $a$ is the only sink and $b$ the only source in $Q_{C}$, there must exist, for each $H_{i}$, an arrow from the unique sink of $Q_{H_{i}}$ to $a$, and an arrow from $b$ to the unique source of $Q_{H_{i}}$. Thus $C=\operatorname{End} T^{\bullet}$ has for quiver

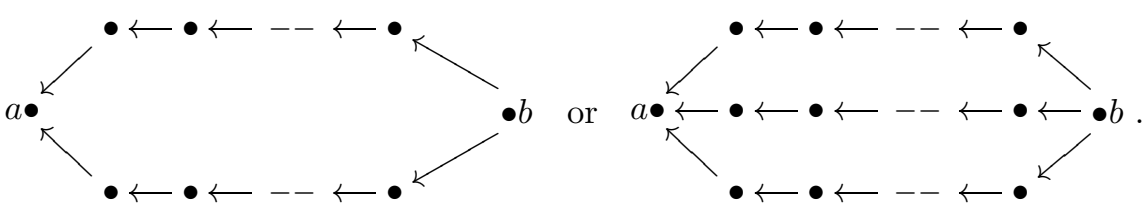

We now determine the possible relations on this quiver. Assume first that there exists a non-zero morphism from $T^{\bullet}(a)$ to $T^{\bullet}(b)$. There exists a hereditary algebra $H^{\prime}$ having a unique simple projective module and a triangle-equivalence $D^{b}\left(\bmod H^{\prime}\right) \cong D^{b}(\bmod H)$ such that $T^{\bullet}(a)$ maps onto the unique simple projective $H^{\prime}$-module. Since there exists a non-zero morphism from $T^{\bullet}(a)$ to $T^{\bullet}(b)$, it follows that $T^{\bullet}(b)$ maps onto an indecomposable $H^{\prime}$-module. But then $T^{\bullet}$ maps onto a tilting $H^{\prime}$-module, and consequently $C=\operatorname{End} T^{\bullet}$ is a tilted algebra. If, on the other hand, there exists no non-zero morphism from $T^{\bullet}(a)$ to $T^{\bullet}(b)$, then, since $C$ is a representation-finite iterated tilted algebra, it is representation-directed (by [7], (IV.3.6), p. 169); hence there exists a zero-relation on each path from $b$ to $a$ in the quiver of $C$. Now, it follows from the structure of the morphisms in the derived category that a relation can only go from the point $b$ to the point $a$. Therefore, $C$ is a one-point extension of a hereditary algebra by a direct sum of (two or three) indecomposable injective modules; hence it is tilted. This completes the proof of our theorem. (We observe that in the last step of the proof, since $C$ is not simply connected, it is in fact iterated tilted of type $\tilde{\mathbb{A}}_{n}$, and it follows easily from the classification in 3 . that $C$ is a one-point extension of a hereditary algebra of type $\mathbb{A}_{3}$ by the direct sum of two simple injective modules). 
3.4. Examples. (a) Our first example shows that Roldán's conjecture, in its original form, is not true. Let $A$ be the algebra given by the quiver

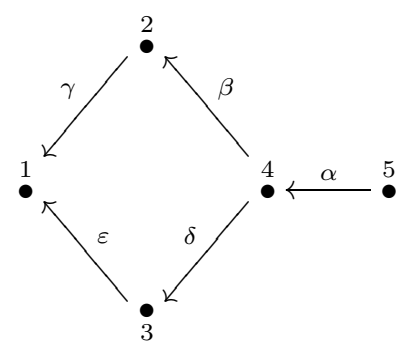

bound by $\beta \gamma=0, \delta \varepsilon=0, \alpha \delta=0$. Then $A$ is a representation-finite iterated tilted algebra of type $\tilde{\mathbb{A}}_{4}$ (see 3 ), it is clearly not tilted. It is easily verified that $A$ has exactly four non-projective and multiplicity-free tilting modules, namely

(i) $T_{A}={ }_{1}^{23} \oplus{ }_{1}^{2} \oplus{ }_{1}^{3} \oplus{ }_{32}^{4} \oplus \underset{2}{5}$,

(ii) $T_{A}={ }_{1}^{2}{ }_{1}^{3}{ }_{1}^{3} \oplus 3 \oplus \underset{32}{4} \oplus \underset{2}{5}$,

(iii) $T_{A}={ }_{1}^{2}{ }_{1}^{3} \underset{1}{2} \oplus 2 \oplus \underset{32}{4} \oplus \underset{2}{5}$,

(iv) $T_{A}={ }_{1}^{2}{ }_{1}^{3} \oplus 2 \oplus 3 \oplus{ }_{32}^{4} \oplus \underset{2}{4}$.

In each case, End $T_{A}$ is representation-infinite (because it contains a representationinfinite hereditary algebra as a full convex subcategory).

(b) Our second example shows that our theorem is not valid for iterated tilted algebras of wild type. Let $B$ be the wild hereditary algebra given by the quiver

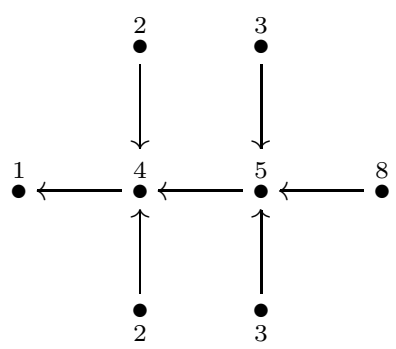

and $C=B / \operatorname{rad}^{2} B$. Then it is easily verified that $C$ is a representation-finite iterated tilted algebra of type $Q_{B}$, and that any going-up tilting series from $C$ to $B$ passes through a non-hereditary representation-infinite algebra.

(c) It is reasonable to ask whether the procedure of the theorem can be achieved by performing first all the APR-tilts, then all the APR- cotilts. This is not possible as is shown by the case of the algebra $D$, which is the one-point coextension of the algebra $A$ of example (a) above by the simple module $S(1)$. 


\section{ACKNOWLEDGEMENTS}

The first and third authors gratefully acknowledge partial support respectively from the Natural Sciences and Engineering Research Council of Canada and CONICET of Argentina. This work was completed during a visit of the second author at the University of Sherbrooke. He would like to thank Shiping Liu for his kind invitation and hospitality.

\section{REFERENCES}

1. Assem, I., Tilting theory - an introduction, in: "Topics in Algebra", vol. 26, Banach Center Publications, PWN, Warsaw, 1990, p. 127-180. MR 93g:16011

2. Assem, I. and Happel, D., Generalized tilted algebras of type $\mathbb{A}_{n}$, vol. 9, Comm. Algebra, 1981, p. 2101-2125. MR 83a:16023a

3. Assem, I. and Skowroński, A., Iterated tilted algebras of type $\tilde{\mathbb{A}}_{n}$, vol. 195, Math. Z, 1987, p. 269-290. MR 88m:16033

4. Assem, I. and Zhang, Y., Endomorphism algebras of exceptional sequences over path algebras of type $\tilde{\mathbb{A}}_{n}$, Colloq. Math. 77 (1998), 271-292. CMP 98:14

5. Auslander, M., Reiten, I. and Smalø,S.O., Representation theory of artin algebras, vol. 36, Cambridge studies in advanced mathematics, Cambridge University Press, 1995. MR 96c:16015

6. Dlab, V. and Ringel, C.M., Indecomposable representations of graphs and algebras, vol. 6, 173, Memoir Amer. Math. Soc, 1976. MR 56:5657

7. Happel, D., Triangulated categories in the representation theory of finite dimensional algebras, London Math. Soc. Lecture Note Series 119, 1988. MR 89e:16035

8. Richard, J., A Morita theory for derived categories, vol. 2, 39, J. London Math. Soc., 1989, p. $436-456$.

9. Ringel, C. M., Tame algebras and integral quadratic forms, vol. 1099, Lecture Notes in Mathematics, Springer-Verlag, Berlin - Heidelberg- New York., 1984. MR 87f:16027

10. Roldán, O., Tilted algebras of types $\tilde{\mathbb{A}}_{n}, \tilde{\mathbb{B}}_{n}, \widetilde{\mathbb{C}}_{n}$ and $\widetilde{\mathbb{B C}}_{n}, \mathrm{Ph}$. D. Thesis, Carleton University (1983).

11. Skowroński, A., Selfinjective algebras of polynomial growth, vol. 285, Math. Ann, 1989, p. 177199. MR 90k:16024

12. Trepode, S.E., A conjectura de Roldán para álgebras inclinadas iteradas de tipo euclideano, Ph. D. Thesis, Universidade de São Paulo, 1995.

13. Trepode, S.E., La conjetura de Roldán en el caso $\tilde{\mathbb{A}}_{n}$, Proc. of the 3rd Conf. of Mathematics Dr. A.A. Monteiro, Bahia Blanca, Argentina, Universidad Nacional del Sur, (1996), p. 51-68. MR 98e:16016

Département de mathématiques et d'informatique, Faculté des sciences, Université DE Sherbrooke, Québec, Canada J1K 2R1

E-mail address: ibrahim.assem@dmi.usherb.ca

Fakultät für Mathematik, TU Chemmitz, PSF 964, D-09107 Chemnitz, Federal RePUBLIC OF GERMANY

E-mail address: happel@mathematik.tu-chemnitz.de

Departamento de Matemáticas, Facultad de Ciencias Exactas y Naturales, UniverSidad nacional de Mar del Plata, Funes 3350, 7600 Mar del Plata, Argentina

E-mail address: strepode@ mdp.edu.ar

Current address: Instituto de Matemáticas, UNAM, Circuito exterior, Cd. Universitaria, México, 04510 D.F., Mexico

E-mail address: sonia@math.unam.mx 